\title{
Psychosocial and Quality of Life in Women Receiving the 21-Gene Recurrence Score Assay: The Impact of Decision Style in Women with Intermediate RS
}

\author{
Nadiyah Sulayman, Elizabeth Spellman, Kristi D. Graves, Beth N. Peshkin, \\ Claudine Isaacs, Marc D. Schwartz, and Suzanne C. O’Neill \\ Department of Oncology, The Fisher Center for Familial Cancer Research, Lombardi Comprehensive Cancer Center, \\ Georgetown University Medical Center, 3300 Whitehaven Street, NW, Suite 4100, Washington, DC 20007, USA \\ Correspondence should be addressed to Suzanne C. O’Neill, sco4@georgetown.edu
}

Received 23 March 2012; Revised 7 June 2012; Accepted 12 June 2012

Academic Editor: Colleen M. McBride

Copyright (C) 2012 Nadiyah Sulayman et al. This is an open access article distributed under the Creative Commons Attribution License, which permits unrestricted use, distribution, and reproduction in any medium, provided the original work is properly cited.

Multigene assays such as the 21-gene recurrence score (RS) quantify risk for recurrence and potential benefit from chemotherapy in early-stage, ER+ breast cancers. Few studies have assessed the impact of testing on patient-reported outcomes such as cancerrelated distress or quality of life. The few studies that have assessed these outcomes do not consider potential modifiers, such as the patients' level of involvement in the treatment decision-making process. In the current study, 81 breast cancer patients who received the RS assay completed cross-sectional surveys. We used linear multiple regression to assess whether test result, decision-making role (passive versus shared/active), and their interaction contributed to current levels of distress, quality of life, and decisional conflict. There were no associations between these variables and test result or decision-making role. However, women who received an intermediate RS and took a passive role in their care reported higher-cancer-related distress and cancer worry and lower quality of life than those who took a shared or active role. These data should be confirmed in prospective samples, as these poorer outcomes could be amenable to intervention.

\section{Introduction}

Improvements in our understanding of the molecular mechanisms of breast cancer progression, diagnosis, and treatment represent a major advance [1]. Genomic profiling of breast tumors increasingly is being used clinically to refine recurrence estimates and guide adjuvant treatment decisions in early-stage, hormone-receptor positive breast cancer [2] and has been integrated into clinical guidelines for this group of patients [3,4]. The 21-gene recurrence score (RS) assay (Oncotype DX; Genomic Health Inc., Redwood City, CA) quantifies risk of recurrence in patients with early stage, estrogen-receptor- (ER-) positive breast cancer treated with tamoxifen. While data have accumulated to support treatment recommendations for the $25 \%$ of women who receive high RS and 50\% who receive low RS, $[5,6]$ recommendations for the remaining quarter of patients with intermediate RS remain less clear pending additional trials, presenting a clinical challenge [7]. Like other cancer treatment decisions which involve more than one appropriate treatment option $[8,9]$, the uncertainty related to this clinical equipoise could require patients to take more active roles in their treatment decision-making than those who receive a high or low RS.

Several studies demonstrate that the RS impacts treatment decisions for physicians and patients [10-14]. Specifically, RS information changes oncologists' chemotherapy treatment recommendations in $25 \%-44 \%$ of cases $[11,13$, 15], usually from combined chemohormonal therapy to hormone therapy alone $[10,11,16]$. However, few studies demonstrate how the RS relates to patient decision-making processes or patient-reported psychosocial and quality of life outcomes. Lo et al. [17] reported that decisional conflict and anxiety decline in the year after receipt of the RS and that quality of life remains stable [17], but they did 
not assess whether outcomes vary by test result or patientrelated factors. Previous research demonstrates that many women who receive their RS do not take part in the treatment decision-making process as they would prefer $[18,19]$. While meeting these decision role preferences has been linked to improved patient outcomes [20,21], breast cancer patients who take a passive role in treatment decisionmaking report lower quality of life and higher distress than women who take an active or shared role, regardless of their preferred decision-making role [22-24]. Therefore, patient involvement in how the RS informs adjuvant care decisions could relate to differential psychosocial and qualityof-life outcomes among tested women. Active or shared involvement with treatment decision-making may be most appropriate in clinical circumstances in which evidence is either incomplete or suggests more than one option could be appropriate $[25,26]$. While breast cancer treatment decisions often involve multiple options and decision points, in the context of the Oncotype RS, active or shared decision making may be particularly salient for women who receive an intermediate RS given the current absence of standard treatment recommendations for this group.

In the present study, we retrospectively evaluated the effect of RS category (i.e., low, intermediate, high RS), decision-making style, and their interaction on patientreported distress, decisional conflict and quality of life in women who had received testing at the time of their diagnosis. We predicted that (1) women who preferred a passive decision-making style would report higher distress and decisional conflict and lower quality of life than women who took an active or shared role in their care and (2) these effects would be stronger in women who had intermediate RS than in women with high or low RS.

\section{Methods}

2.1. Study Population. Female participants were recruited from 2009-2010 and had been treated at Lombardi Comprehensive Cancer Center from 2005-2009. We identified patients who had received testing through pathology record systems that track all tests ordered at Lombardi. Eligibility included having been tested with the 21-gene RS test, having completed chemotherapy treatment, and not having a recurrence of their primary breast cancer or a second cancer. In addition, participants could not have other major comorbid disease or participate in clinical trials that could have impacted treatment decision making among women with an RS, as these circumstances would have removed the treatment decision from the patient and their physician. Only women with valid contact information were considered eligible. The study was approved by the Institutional Review Board of Georgetown University. All participants provided written informed consent.

Eligible women received a mailed survey, consent and HIPAA documents, a letter of invitation from their attending physician, and a self-addressed stamped envelope in which to return the study documents. They also received a selfaddressed stamped postcard by which they could indicate their interest in declining the study. We identified 128

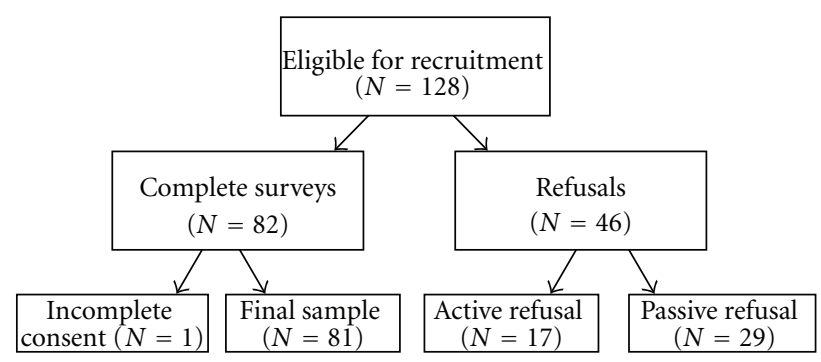

Figure 1: Participant recruitment and enrollment.

potentially eligible women diagnosed with early-stage, ER+ breast cancer who had received the 21-gene RS test results confirmed via chart review. Of these, 46 (36\%) refused participation (17 active and 29 passive refusals); 1 returned her questionnaire without a signed consent form and did not respond to requests to complete the consent. Thus, our final sample of 81 women who received testing and completed questionnaires and consents represents $63 \%$ of the eligible sample (Figure 1). While we were unable to obtain clinical characteristics from women who refused participation, our final sample was similar in race/ethnicity to our clinic population (70\% white).

\subsection{Measures}

2.2.1. Participants' Characteristics. We assessed age, education, race, marital, and financial status. A trained research assistant performed chart reviews to obtain the following: RS, tumor stage and grade, receptor status, primary therapy, and adjuvant therapy. We computed participants' 10-year risk for recurrence using a validated tool (Adjuvant! Online) that provides a quantitative risk estimate using clinical parameters [27, 28]. In addition to chart review, we also assessed RS and treatments received via self-report. Given that all patients were recruited during the clinical trial to determine treatment recommendations for women with intermediate RS (TailoRx), we categorized RS using the trial guidelines: low $(<11)$, intermediate $(11-25)$, and high $(>25)$.

2.2.2. Decision Style. We used the Control Preferences Scale $[29,30]$ to assess patients' preferences for shared medical decision making. The scale is composed of five statements which are separated into passive "I preferred that my doctor make the decision about treatment; I preferred that my doctor make the decision about treatment, but strongly considers my opinion," shared "I preferred that my doctor and I make the decision about treatment together on an equal basis" and active "I preferred that I make the decision about treatment, but strongly consider the doctor's opinion; I preferred that I make the decision about treatment" decision styles. Patients were asked "which of these best describes your way of making a decision about your cancer treatment?"

2.2.3. Cancer-Related Distress. We used the 15 -item Impact of Event Scale (IES) [31] to measure cancer-specific distress. The IES measures intrusive and avoidant thoughts/behaviors 
associated with a specific stressor. Items are scored on a 4point scale (not at all-often), indicating how frequently each thought/behavior occurred during the past seven days in regards to their cancer diagnosis. This scale had excellent internal consistency $(\alpha=.93)$. A score greater than 19 indicates high distress [31].

2.2.4. Cancer Worry. We assessed how often the participant worried about her risk of recurrence during the past two weeks, measured on a 4-point Likert scale (not at all-all of the time) [32].

2.2.5. Quality of Life. We measured quality of life with the FACT-B [33], a well-validated and frequently used measure of health-related quality of life in patients with breast cancer. It consists of the FACT-General (FACT-G) and the 9-item Breast Cancer Subscale. Patients indicated how true each statement was for them in the past seven days measured on a 5 -point Likert scale $(0=$ not at all to $4=$ very $m u c h)$, with higher scores indicating better quality of life. This scale demonstrated excellent internal consistency $(\alpha=.92)$.

2.2.6. Decisional Conflict. We assessed decisional conflict with the Decisional Conflict Scale (DCS) [34], which consists of 16 items measured on a 5-point Likert scale $(1=$ strongly agree to $5=$ strongly disagree). Patients were asked to think about the choice they had made about their breast cancer treatment. The DCS measures uncertainty about the decision (3 items), feeling uninformed about the decision (3 items), feeling unsupported in decision making (3 items), feeling unclear about values ( 3 items), and the perceived quality of the decision (4 items). Items were averaged so that higher scores indicated higher decisional conflict. The measure demonstrated good internal reliability $(\alpha=.89)$. A score of 2 or above is considered high decisional conflict.

2.3. Data Analysis. We calculated descriptive statistics for all sociodemographic, treatment-related, psychosocial, and quality of life variables. We assessed bivariate associations between sociodemographics and treatment-related variables, as well as psychosocial, and quality of life outcomes. In multivariate analyses, we adjusted for all variables with bivariate associations of $P<.10$. We used multiple linear regression with hierarchical variable entry, entering covariates on Step 1, decision style on Step 2, RS category on Step 3, and their interaction in the final step. Data were analyzed using SPSS 19.0. With a sample of 81 participants, we were powered to find effects of .17 for our main effects and .26 for our interaction effects.

\section{Results}

3.1. Participant Characteristics. Mean scores and percentages are shown in Table 1. Participants were, on average, 19 months from diagnosis and testing (range $=6-$ 64 months). All women who received high or low RS results received result-concordant treatment, with higher risk patients receiving chemohormonal therapy and lower risk patients receiving hormonal therapy only. Among patients with intermediate RS, 38 (75\%) received hormonal therapy only and $13(25 \%)$ received combined therapy. The latter group had marginally higher risk according to Adjuvant! scores (26.6 versus $22.3, t(44)=1.72, P=.09$ ). Most women indicated that they preferred a shared $(36 \%)$ or active $(49 \%)$ decision-making style in their treatment for breast cancer. The only sociodemographic or treatment variable related to our outcomes was race. White women had significantly higher cancer worry $(t(79)=2.05, P=.04)$ and lower quality of life $(t(79)=-2.00, P=.05)$ compared to others. We adjusted for race in subsequent models. Importantly, neither time since diagnosis nor receipt of chemotherapy was related to any of our outcomes.

3.2. Levels of Distress, Decisional Conflict, and Quality of Life. Women reported moderate levels of cancer-related distress $(M=19.10, \mathrm{SD}=17.50)$, cancer worry $(M=1.70, \mathrm{SD}=0.80)$, and decisional conflict $(M=1.70, \mathrm{SD}=0.50)$. Though overall values indicate adequate global functioning in this sample, $30 \%$ of women reported problematic levels of decisional conflict (scores $\geq 2$ ) and $38.7 \%$ of women reported high levels of cancer-related distress (scores $>19$ ) [35].

3.3. Association of Decision Style and RS with Distress and Decisional Conflict. We conducted separate hierarchical multiple regression analyses for cancer-related distress, worry about recurrence, and decisional conflict. After controlling for race on Step 1, we entered decision style on Step 2, RS category (intermediate versus low/high) on Step 3, and their interaction in the final step (Table 2). Neither decision style on Step 2 nor RS categories on Step 3 were associated with cancer-related distress or worry about recurrence. However, in the final model on Step 4, the interaction between decision style and RS was associated with cancer-related distress $\left(\Delta R^{2}=.12, P=.002\right)$ and worry about recurrence $\left(\Delta R^{2}=.05, P=.04\right)$. We further assessed these significant interactions with simple effects analysis. As displayed in Figure 2, among women who preferred an active/shared role in their care, RS was unrelated to cancer-related distress. However, among women who preferred a passive role, results were poorer for women with intermediate RS than high/low RS. Specifically, among women with an intermediate RS, a passive versus shared/active decision style was related to higher cancer-related distress (38.20 versus $17.40 ; t(49)=$ $2.75, P=.008)$. This simple effects analysis was not significant for worry about recurrence ( 1.70 versus 1.60 , $t(49)=.30, P=.77)$. In our model for decisional conflict, those who preferred a passive decision style reported significantly higher decisional conflict on Step $2\left(\Delta R^{2}=.06\right.$, $P=.03)$. However, this was not significant in the final model. Neither RS nor the decision style $\mathrm{x}$ RS interaction was significantly associated with decisional conflict.

3.4. Association of Decision Style and RS with Quality of Life. We also tested the association between decision style, test result, and their interaction on quality of life. Neither decision style on Step 2 nor RS categories on Step 3 were 
TABLE 1: Sociodemographic and medical characteristics $(N=81)$.

\begin{tabular}{|c|c|c|}
\hline Sociodemographics & $N(\%)$ & $M(\mathrm{SD})$ \\
\hline Age & & $54.35(9.18)$ \\
\hline \multicolumn{3}{|l|}{ Education } \\
\hline$<$ College degree & $22(27)$ & \\
\hline College degree & $19(23)$ & \\
\hline Graduate/professional training & $40(50)$ & \\
\hline \multicolumn{3}{|l|}{ Race } \\
\hline Caucasian & $62(77)$ & \\
\hline Non-Caucasian & $19(23)$ & \\
\hline \multicolumn{3}{|l|}{ Marital status } \\
\hline Married/partner & $50(62)$ & \\
\hline Single/widow/divorced & $31(38)$ & \\
\hline \multicolumn{3}{|l|}{ Annual household income } \\
\hline$<\$ 50,000$ & $14(17)$ & \\
\hline$\$ 50,000-100,000$ & $10(12)$ & \\
\hline$>\$ 100,000$ & $45(56)$ & \\
\hline Refused/missing & $12(15)$ & \\
\hline \multicolumn{3}{|l|}{ Treatments received } \\
\hline \multicolumn{3}{|l|}{ Surgery } \\
\hline Lumpectomy & $48(59)$ & \\
\hline Unilateral mastectomy & $17(21)$ & \\
\hline Bilateral mastectomy & $16(20)$ & \\
\hline \multicolumn{3}{|l|}{ Chemotherapy } \\
\hline Yes & $24(30)$ & \\
\hline No & $57(70)$ & \\
\hline \multicolumn{3}{|l|}{ Radiation } \\
\hline Yes & $24(30)$ & \\
\hline No & $57(70)$ & \\
\hline \multicolumn{3}{|l|}{ Tamoxifen } \\
\hline Yes & $47(58)$ & \\
\hline No & $34(42)$ & \\
\hline \multicolumn{3}{|l|}{ Aromatase inhibitors } \\
\hline Yes & $32(40)$ & \\
\hline No & $48(59)$ & \\
\hline Do not know & $1(1)$ & \\
\hline \multicolumn{3}{|l|}{ Recurrence Score $\times$ Treatment } \\
\hline Low-hormonal therapy & $19(23)$ & \\
\hline Intermediate-hormonal therapy & $38(47)$ & \\
\hline Intermediate-chemohormonal therapy & $13(16)$ & \\
\hline High-chemohormonal therapy & $11(14)$ & \\
\hline \multicolumn{3}{|l|}{ Psychosocial and quality of life variables } \\
\hline \multicolumn{3}{|l|}{ Decision style } \\
\hline Passive & $12(15)$ & \\
\hline Shared & $29(36)$ & \\
\hline Active & $40(49)$ & \\
\hline Cancer-related distress (IES) & & $19.06(17.51)$ \\
\hline Decisional conflict & & $1.69(.51)$ \\
\hline Worry about recurrence & & $1.65(.83)$ \\
\hline Quality of life (FACT-B) & & $111.75(18.85)$ \\
\hline
\end{tabular}


TABLE 2: Multivariate regression analysis of relationship between decision style, RS, and patient-reported outcomes.

\begin{tabular}{lcccccccccccc}
\hline & \multicolumn{3}{c}{ FACT-B } & \multicolumn{3}{c}{ IES } & \multicolumn{3}{c}{ DCS } & \multicolumn{3}{c}{ Cancer worry } \\
& $\Delta R^{2}$ & $\Delta F$ & Final $\beta$ & $\Delta R^{2}$ & $\Delta F$ & Final $\beta$ & $\Delta R^{2}$ & $\Delta F$ & Final $\beta$ & $\Delta R^{2}$ & $\Delta F$ & Final $\beta$ \\
\hline Race & 0.04 & 3.03 & -0.20 & 0.02 & 1.86 & 0.17 & 0.02 & 1.84 & 0.11 & 0.05 & $4.06^{*}$ & $0.27^{*}$ \\
Decision style $^{1}$ & 0.02 & 1.25 & $-0.31^{*}$ & 0.01 & 0.67 & $0.40^{* *}$ & 0.06 & $4.66^{*}$ & 0.25 & 0.02 & 1.93 & 0.02 \\
RS test result $^{2}$ & 0.01 & 0.06 & -0.13 & 0.01 & 0.35 & 0.08 & 0.01 & 0.45 & -0.07 & 0.01 & 0.30 & 0.16 \\
Decision style $\times$ test result & 0.05 & $4.14^{*}$ & $0.31^{*}$ & 0.12 & $10.49^{* *}$ & $-0.49^{* *}$ & 0.00 & 0.01 & -0.01 & 0.05 & $4.23^{*}$ & $-0.31^{*}$ \\
\hline
\end{tabular}

${ }^{1}$ Passive versus shared/active. ${ }^{2}$ Intermediate RS versus high/low RS.

${ }^{*} P<.05 ;{ }^{* *} P<.01$.

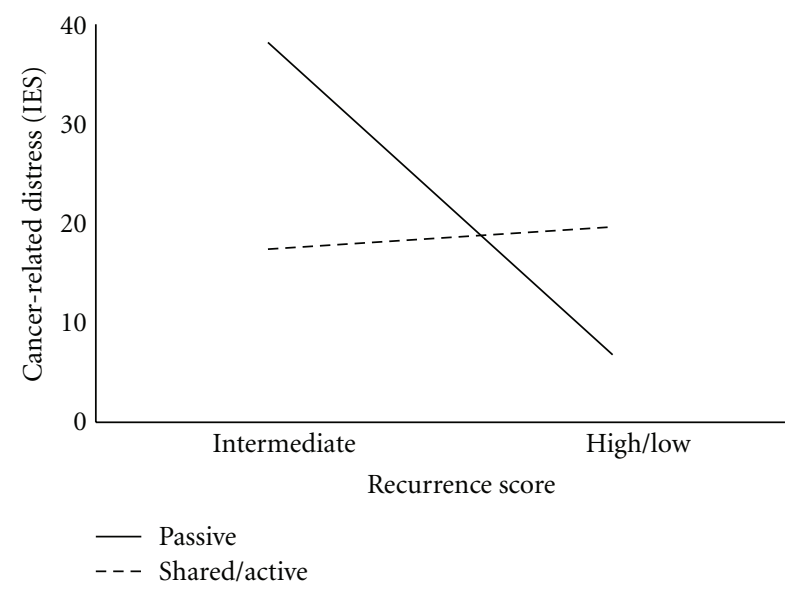

FIGURe 2: Cancer-related distress (IES) by RS (intermediate versus high/low) and decision style (passive versus shared/active).

associated with quality of life. However, on Step 4, the interaction between decision style and RS was associated with quality of life $\left(\Delta R^{2}=.05, P=.04\right)$. Again, our simple effects analysis demonstrated that while there were no significant differences among women who preferred an active/shared role in their care, among women who preferred a passive role, those with intermediate RS reported poorer quality of life than those high/low RS (Figure 3). Specifically, among women with an intermediate RS, those who preferred a passive role in their care reported significantly lower quality of life than those who took an active/shared role (97.60 versus $114.40 ; t(49)=-2.27, P=.03)$.

\section{Discussion}

The 21-gene RS impacts adjuvant treatment decisions of patients and their providers [10-14]. In the present study, we found that women who receive their RS have levels of quality of life and distress comparable to other women who are a year or more from being diagnosed and treated for early-stage breast cancer and make RS-congruent treatment decisions $[17,36,37]$. Further, we found no evidence that these associations vary based on the risk of recurrence indicated by the RS or the treatment received. However, in this sample of tested women, a passive decision role preference was associated with higher distress and lower quality of life. This is especially true for women who received an intermediate RS. In addition, like the broader population of women diagnosed

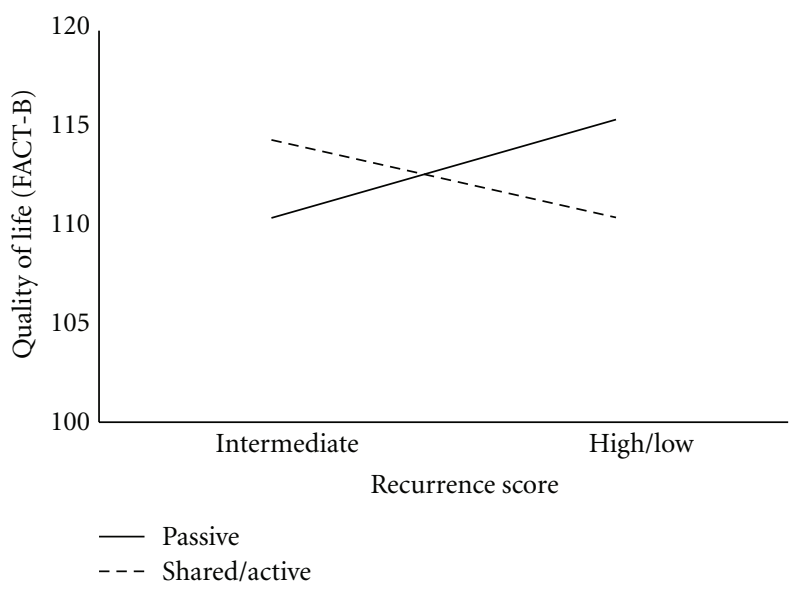

FIGURE 3: Quality of life (FACT-B) by RS (intermediate versus high/low) and decision style (passive versus shared/active).

with breast cancer, a substantial number-more than onethird-of women continue to experience significant distress, even a year or more after diagnosis.

The present study extends prior results by demonstrating that while women who receive RS testing, as a whole, do well, there is significant variability in outcomes as a result of both test result received and the level of involvement these women take in their treatment decisions. Indeed, $40 \%$ of the sample reported problematic levels of cancer-related distress, a substantial number considering these women were several months removed from their active treatment. This is similar to previous work on women who receive $B R C A 1 / 2$ mutation testing. Several systematic reviews demonstrate that, as a whole, genetic counseling and testing for breast cancer susceptibility do not result in significant psychological distress or decrements in quality of life for this population of patients as a whole [38-40]. However, an extensive literature, including our own work, demonstrates that some women, such as those with high pretesting distress, a significant family history of the disease, a high expectation for carrying a deleterious mutation, and difficulty with uncertain health information, are at risk for long-term distress [37, 41]. Similarly, most women who receive their RS may adjust well, but a subgroup of women may be at risk for poorer outcomes and in need of additional support. This question deserves additional research in larger, more representative samples.

Long-standing trends in health care call for patients to be more involved in their care [42]. Patient involvement and 
integration with treatment decision making may be most appropriate in clinical circumstances in which evidence is either incomplete or suggests more than one option could be appropriate $[25,26]$. Previous studies of women with breast cancer show that quality of life and satisfaction are associated with active or shared involvement in care decisions [21, 23, $43,44]$. The clinical equipoise and uncertainties associated with an intermediate RS could mean that treatment decisions rely more on women's treatment-related values and preferences in combination with standard clinical criteria and the physician's clinical judgment. Importantly, the receipt of chemotherapy among women with intermediate RS was marginally associated with the patient's Adjuvant! Online score, suggesting that clinical factors are an important part, but not the only consideration, in treatment decisions. This underscores the multifaceted nature of treatment decisions in women with intermediate RS at this time [7].

The uncertainties associated with intermediate RS could make the need for active or shared decision making more apparent. Genomic medicine often makes the uncertainties that are ever-present in medical decisions more obvious to patients, as risks often are communicated as probabilities and on continua $[8,45]$. The intermediate RS is one prominent example of this, but it is not the only one and there are likely to be many others. How patients and providers communicate about these uncertainties and come to treatment decisions will likely impact the benefit that patients ultimately will gain from these new technologies. Women in this group may benefit from decision support interventions aimed at fostering shared decision making and providing the tools to do so $[46,47]$ or interventions to promote coping and reduce distress in the face of clinical uncertainty. Interestingly, despite $30 \%$ of our sample indicating problematic levels of decisional conflict, neither test result, decision style nor their interaction was associated with this variable. Our crosssectional data do not allow us to determine whether these null findings are a result of being removed in time from the treatment decision, measurement issues, or another explanation. Future prospective, longitudinal research should examine this issue and how it would influence the development of interventions for this population.

This study has several limitations, including a retrospective, cross-sectional design and a relatively small sample limited to women who had completed treatment for breast cancer. Specifically, participants may not accurately remember their decision role preference. However, previous research has found strong associations between these retrospective perceptions and those measured at the time of care [48] and retrospective methods are used frequently in cancer decision-making research $[49,50]$. Future research should follow women prospectively from the time of diagnosis and RS testing. Ideally, this would occur before they receive their RS results and make definite treatment decisions in order to prospectively assess the impact of the testing and treatment decision making process on long-term patientreported outcomes. We were also limited by lack of an untested control group to which to compare our outcomes. At the time the study was implemented, testing had become the standard of care, limiting the ability to make this comparison [4]. Another limitation includes our use of a single site at a comprehensive cancer center and a patient population with a relatively high self-reported income. The patient population and clinical encounters captured in the current study may not fully reflect those in other settings. This could also be impacted by our relatively low response rate. Our use of a mailed survey as a means of recruitment may also have resulted in a lower rate of recruitment than if we have been able to recruit women in person. Finally, all of the women in the study had lymph node-negative disease. As testing becomes more common among women with limited node involvement $[51,52]$, the need for greater active or shared decision making for these women may expand as well.

In conclusion, this study underscores the important role that patient decision-making style plays for women who receive $\mathrm{RS}$ as part of their diagnosis and treatment process. This is particularly true for those with intermediate RS. These results hold greater uncertainty, and consequently, might require more input from patients to clarify the values and preferences they hold regarding their care. Our results should be replicated in larger, prospective, multicenter study of women who have received this testing. Future research also should determine the best means to assist these patients in participating in their care decisions.

\section{Acknowledgments}

This study and paper preparation were supported by Grants from the American Cancer Society (97-152-04 to Georgetown University and MRSG-10-110-01-CPPB to S. C. O'Neill). The authors thank the physicians and staff of the Georgetown Lombardi Comprehensive Cancer Center for their assistance during the study. They especially thank Dale Gibson in the Department of Pathology for his help in developing our recruitment process. Most importantly, they thank the women who participated in this study.

\section{References}

[1] O. I. Olopade, T. A. Grushko, R. Nanda, and D. Huo, "Advances in breast cancer: pathways to personalized medicine," Clinical Cancer Research, vol. 14, no. 24, pp. 7988-7999, 2008.

[2] L. Harris, H. Fritsche, R. Mennel et al., "American society of clinical oncology 2007 update of recommendations for the use of tumor markers in breast cancer," Journal of Clinical Oncology, vol. 25, no. 33, pp. 5287-5312, 2007.

[3] N. Ben-Baruch, A. Hammerman, S. Klang, and N. Liebermann, "A prospective study of the impact of the 21-gene recurrence score assay on treatment decisions in N-, ER+ early stage breast cancer patients," Journal of Clinical Oncology, vol. 25, no. 18, abstract 1108, 2007.

[4] National Comprehensive Cancer Network, NCCN Clinical Practice Guidelines in Oncology. Breast Cancer. Version 1, 2012, http://www.nccn.org/professionals/physician_gls/pdf/ breast.pdf.

[5] E. P. Mamounas, G. Tang, B. Fisher et al., "Association between the 21-gene recurrence score assay and risk of locoregional recurrence in node-negative, estrogen receptor-positive breast 
cancer: results from NSABP B-14 and NSABP B-20," Journal of Clinical Oncology, vol. 28, no. 10, pp. 1677-1683, 2010.

[6] S. Paik, G. Tang, S. Shak et al., "Gene expression and benefit of chemotherapy in women with node-negative, estrogen receptor-positive breast cancer," Journal of Clinical Oncology, vol. 24, no. 23, pp. 3726-3734, 2006.

[7] J. A. Sparano and S. Paik, "Development of the 21-gene assay and its application in clinical practice and clinical trials," Journal of Clinical Oncology, vol. 26, no. 5, pp. 721-728, 2008.

[8] A. H. Pieterse, I. Henselmans, H. C. J. M. de Haes, C. C. E. Koning, E. D. Geijsen, and E. M. A. Smets, "Shared decision making: prostate cancer patients' appraisal of treatment alternatives and oncologists' eliciting and responding behavior, an explorative study," Patient Education and Counseling, vol. 85, no. 3, pp. e251-e259, 2011.

[9] A. Sidana, D. J. Hernandez, Z. Feng et al., "Treatment decisionmaking for localized prostate cancer: what younger men choose and why," Prostate, vol. 72, no. 1, pp. 58-64, 2012.

[10] J. Albanell, A. Gonzalez, M. Ruiz-Borrego et al., "Prospective transGEICAM study of the impact of the 21-gene Recurrence Score assay and traditional clinicopathological factors on adjuvant clinical decision making in women with estrogen receptor-positive $(\mathrm{ER}+)$ node-negative breast cancer," Annals of Oncology, vol. 23, no. 3, pp. 625-631, 2012.

[11] J. Asad, A. F. Jacobson, A. Estabrook et al., "Does oncotype DX recurrence score affect the management of patients with earlystage breast cancer?" American Journal of Surgery, vol. 196, no. 4, pp. 527-529, 2008.

[12] D. B. Geffen, S. Abu-Ghanem, N. Sion-Vardy et al., "The impact of the 21-gene recurrence score assay on decision making about adjuvant chemotherapy in early-stage estrogenreceptor-positive breast cancer in an oncology practice with a unified treatment policy," Annals of Oncology, vol. 22, no. 11, pp. 2381-2386, 2011.

[13] L. R. Henry, A. Stojadinovic, S. M. Swain, S. Prindiville, R. Cordes, and P. W. Soballe, "The influence of a gene expression profile on breast cancer decisions," Journal of Surgical Oncology, vol. 99, no. 6, pp. 319-323, 2009.

[14] J. F. Partin and E. P. Mamounas, "Impact of the 21-gene recurrence score assay compared with standard clinicopathologic guidelines in adjuvant therapy selection for node-negative, estrogen receptor-positive breast cancer," Annals of Surgical Oncology, vol. 18, no. 12, pp. 3399-3406, 2011.

[15] R. Oratz, D. Paul, A. L. Cohn, and S. M. Sedlacek, "Impact of a commercial reference laboratory test recurrence score on decision making in early-stage breast cancer," Journal of Oncology Practice, vol. 3, no. 4, pp. 182-186, 2007.

[16] J. A. Rayhanabad, L. A. Difronzo, P. I. Haigh, and L. Romero, "Changing paradigms in breast cancer management: introducing molecular genetics into the treatment algorithm," American Surgeon, vol. 74, no. 10, pp. 887-890, 2008.

[17] S. S. Lo, P. B. Mumby, J. Norton et al., "Prospective multicenter study of the impact of the 21-gene recurrence score assay on medical oncologist and patient adjuvant breast cancer treatment selection," Journal of Clinical Oncology, vol. 28, no. 10, pp. 1671-1676, 2010.

[18] N. T. Brewer, A. R. Richman, J. T. Defrank, V. F. Reyna, and L. A. Carey, "Improving communication of breast cancer recurrence risk," Breast Cancer Research and Treatment, vol. 133, no. 2, pp. 553-561, 2012.

[19] J. P. Tzeng, D. Mayer, A. R. Richman et al., "Women's experiences with genomic testing for breast cancer recurrence risk," Cancer, vol. 116, no. 8, pp. 1992-2000, 2010.
[20] R. Brown, P. Butow, M. Wilson-Genderson, J. Bernhard, K. Ribi, and I. Juraskova, "Meeting the decision-making preferences of patients with breast cancer in oncology consultations: impact on decision-related outcomes," Journal of Clinical Oncology, vol. 30, no. 8, pp. 857-862, 2012.

[21] M. Gattellari, P. N. Butow, and M. H. N. Tattersall, "Sharing decisions in cancer care," Social Science and Medicine, vol. 52, no. 12, pp. 1865-1878, 2001.

[22] M. R. Andersen, D. J. Bowen, J. Morea, K. D. Stein, and F. Baker, "Involvement in Decision-Making and Breast Cancer Survivor Quality of Life," Health Psychology, vol. 28, no. 1, pp. 29-37, 2009.

[23] T. F. Hack, L. F. Degner, P. Watson, and L. Sinha, "Do patients benefit from participating in medical decision making? Longitudinal follow-up of women with breast cancer," PsychoOncology, vol. 15, no. 1, pp. 9-19, 2006.

[24] T. F. Hack, T. Pickles, J. D. Ruether et al., "Predictors of distress and quality of life in patients undergoing cancer therapy: impact of treatment type and decisional role," PsychoOncology, vol. 19, no. 6, pp. 606-616, 2010.

[25] "Taking shared decision making more seriously," Lancet, vol. 377, no. 9768, p. 784, 2011.

[26] A. Barratt, "Evidence based medicine and shared decision making: the challenge of getting both evidence and preferences into health care," Patient Education and Counseling, vol. 73, no. 3, pp. 407-412, 2008.

[27] P. M. Ravdin, L. A. Siminoff, G. J. Davis et al., "Computer program to assist in making decisions about adjuvant therapy for women with early breast cancer," Journal of Clinical Oncology, vol. 19, no. 4, pp. 980-991, 2001.

[28] L. A. Siminoff, N. H. Gordon, P. Silverman, T. Budd, and P. A. Ravdin, "A decision aid to assist in adjuvant therapy choices for breast cancer," Psycho-Oncology, vol. 15, no. 11, pp. 10011013, 2006.

[29] L. F. Degner and C. A. Russell, "Preferences for treatment control among adults with cancer," Research in Nursing \& Health, vol. 11, no. 6, pp. 367-374, 1988.

[30] L. F. Degner, L. J. Kristjanson, D. Bowman et al., "Information needs and decisional preferences in women with breast cancer," Journal of the American Medical Association, vol. 277, no. 18, pp. 1485-1492, 1997.

[31] M. Horowitz, N. Wilner, and W. Alvarez, "Impact of event scale: a measure of subjective stress," Psychosomatic Medicine, vol. 41, no. 3, pp. 209-218, 1979.

[32] C. Lerman, B. Trock, B. K. Rimer, C. Jepson, D. Brody, and A. Boyce, "Psychological side effects of breast cancer screening," Health Psychology, vol. 10, no. 4, pp. 259-267, 1991.

[33] M. J. Brady, D. F. Cella, F. Mo et al., "Reliability and validity of the functional assessment of cancer therapy- breast qualityof-life instrument," Journal of Clinical Oncology, vol. 15, no. 3, pp. 974-986, 1997.

[34] A. M. O'Connor, "Validation of a decisional conflict scale," Medical Decision Making, vol. 15, no. 1, pp. 25-30, 1995.

[35] M. Horowitz, "Stress response syndromes and their treatment," in Handbook of Stress: Theoretical and Clinical Aspects, L. Goldberger and S. Breznitz, Eds., pp. 209-218, Free Press, New York, NY, USA, 1982.

[36] N. E. Avis, S. Crawford, and J. Manuel, "Quality of life among younger women with breast cancer," Journal of Clinical Oncology, vol. 23, no. 15, pp. 3322-3330, 2005.

[37] S. C. O’Neill, C. Rini, R. E. Goldsmith, H. Valdimarsdottir, L. H. Cohen, and M. D. Schwartz, "Distress among women receiving uninformative BRCA1/2 results: 12-month 
outcomes," Psycho-Oncology, vol. 18, no. 10, pp. 1088-1096, 2009.

[38] D. Braithwaite, J. Emery, F. Walter, A. T. Prevost, and S. Sutton, "Psychological impact of genetic counseling for familial cancer: a systematic review and meta-analysis," Journal of the National Cancer Institute, vol. 96, no. 2, pp. 122-133, 2004.

[39] J. G. Hamilton, M. Lobel, and A. Moyer, "Emotional distress following genetic testing for hereditary breast and ovarian cancer: a meta-analytic review," Health Psychology, vol. 28, no. 4, pp. 510-518, 2009.

[40] J. S. Hilgart, B. Coles, and R. Iredale, "Cancer genetic risk assessment for individuals at risk of familial breast cancer," Cochrane Database of Systematic Reviews, no. 2, Article ID CD003721, 2007.

[41] C. Rini, S. C. O’Neill, H. Valdimarsdottir et al., "Cognitive and emotional factors predicting decisional conflict among high-risk breast cancer survivors who receive uninformative BRCA1/2 results," Health Psychology, vol. 28, no. 5, pp. 569$578,2009$.

[42] S. Brownlee, J. E. Wennberg, M. J. Barry, E. S. Fisher, D. C. Goodman, and J. P. W. Bynum, "Improving patient decisionmaking in health care: a 2011 Dartmouth Atlas report highlighting Minnesota," http://www.dartmouthatlas.org/ downloads/reports/Decision_making_report_022411.pdf.

[43] S. J. Katz, P. M. Lantz, N. K. Janz et al., "Patient involvement in surgery treatment decisions for breast cancer," Journal of Clinical Oncology, vol. 23, no. 24, pp. 5526-5533, 2005.

[44] P. M. Lantz, N. K. Janz, A. Fagerlin et al., "Satisfaction with surgery outcomes and the decision process in a populationbased sample of women with breast cancer," Health Services Research, vol. 40, no. 3, pp. 745-767, 2005.

[45] D. H. Lea, K. A. Kaphingst, D. Bowen, I. Lipkus, and D. W. Hadley, "Communicating genetic and genomic information: health literacy and numeracy considerations," Public Health Genomics, vol. 14, no. 4-5, pp. 279-289, 2011.

[46] S. T. Hawley, P. M. Lantz, N. K. Janz et al., "Factors associated with patient involvement in surgical treatment decision making for breast cancer," Patient Education and Counseling, vol. 65, no. 3, pp. 387-395, 2007.

[47] J. F. Waljee, M. A. M. Rogers, and A. K. Alderman, "Decision aids and breast cancer: do they influence choice for surgery and knowledge of treatment options?" Journal of Clinical Oncology, vol. 25, no. 9, pp. 1067-1073, 2007.

[48] H. S. Gong, J. K. Huh, J. H. Lee, M. B. Kim, M. S. Chung, and G. H. Baek, "Patients' preferred and retrospectively perceived levels of involvement during decision-making regarding carpal tunnel release," Journal of Bone and Joint Surgery Series A, vol. 93, no. 16, pp. 1527-1533, 2011.

[49] S. T. Hawley, J. J. Griggs, A. S. Hamilton et al., "Decision involvement and receipt of mastectomy among racially and ethnically diverse breast cancer patients," Journal of the National Cancer Institute, vol. 101, no. 19, pp. 1337-1347, 2009.

[50] C. N. Lee, Y. Chang, N. Adimorah et al., "Decision making about surgery for early-stage breast cancer," Journal of the American College of Surgeons, vol. 214, no. 1, pp. 1-10, 2012.

[51] M. Dowsett, J. Cuzick, C. Wale et al., "Prediction of risk of distant recurrence using the 21-gene recurrence score in node-negative and node-positive postmenopausal patients with breast cancer treated with anastrozole or tamoxifen: a TransATAC study," Journal of Clinical Oncology, vol. 28, no. 11, pp. 1829-1834, 2010.
[52] R. Oratz, B. Kim, C. Chao et al., "Physician survey of the effect of the 21-gene recurrence score assay results on treatment recommendations for patients with lymph node-positive, estrogen receptor-positive breast cancer," Journal of Oncology Practice, vol. 7, no. 2, pp. 94-99, 2011. 


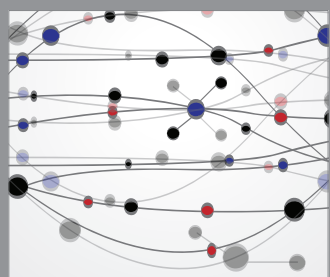

The Scientific World Journal
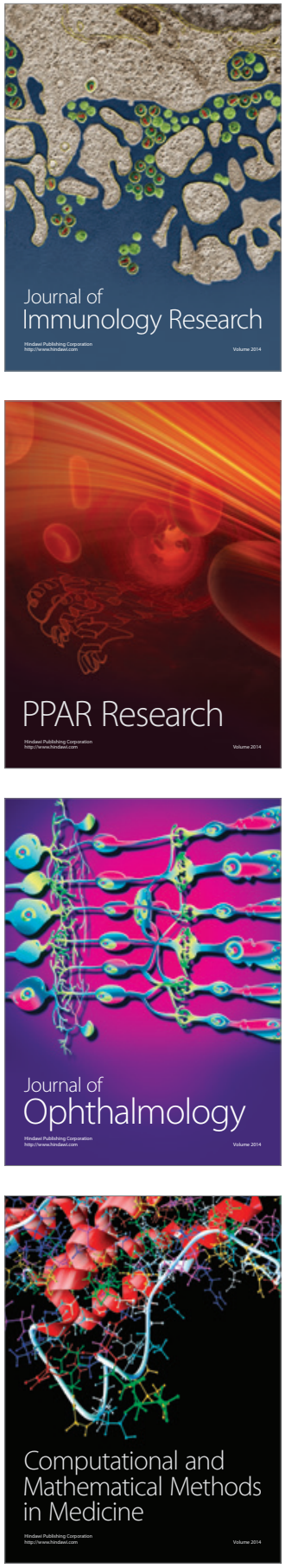

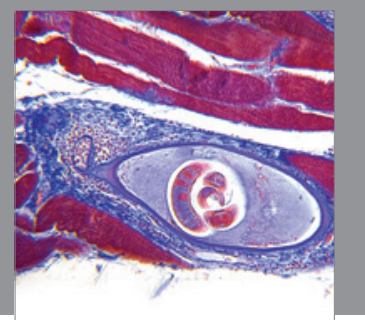

Gastroenterology

Research and Practice
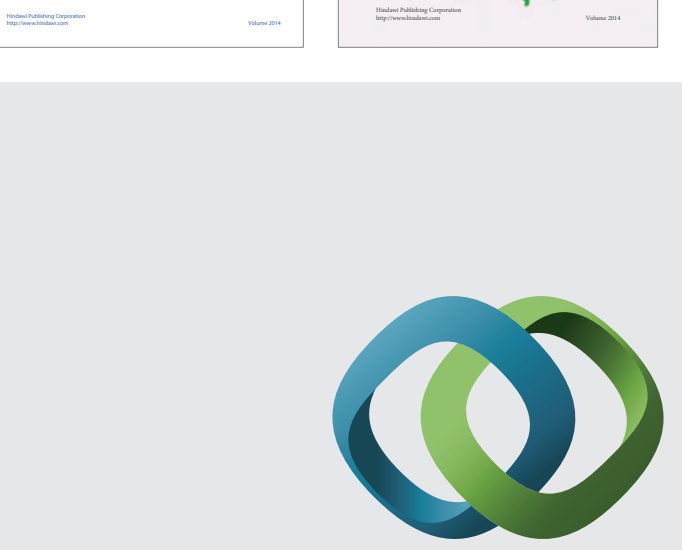

\section{Hindawi}

Submit your manuscripts at

http://www.hindawi.com
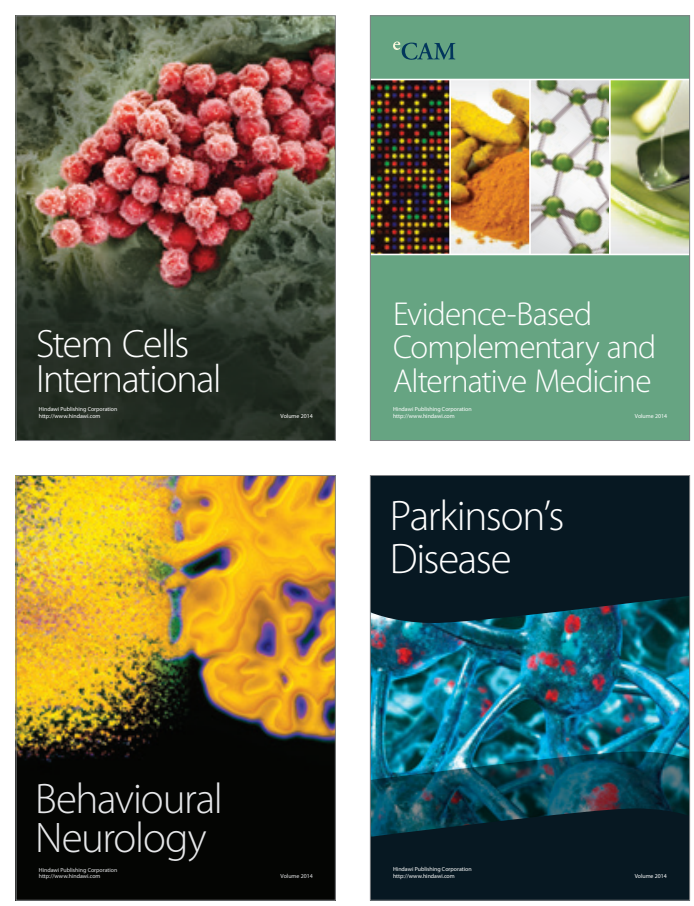

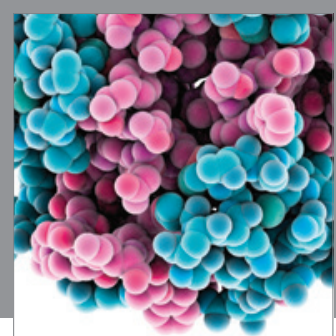

Journal of
Diabetes Research

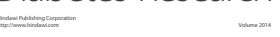

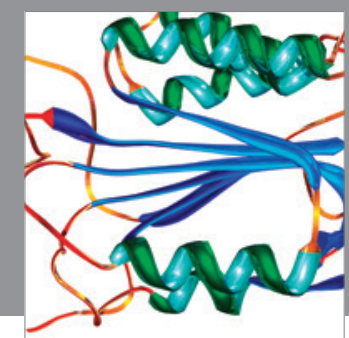

Disease Markers
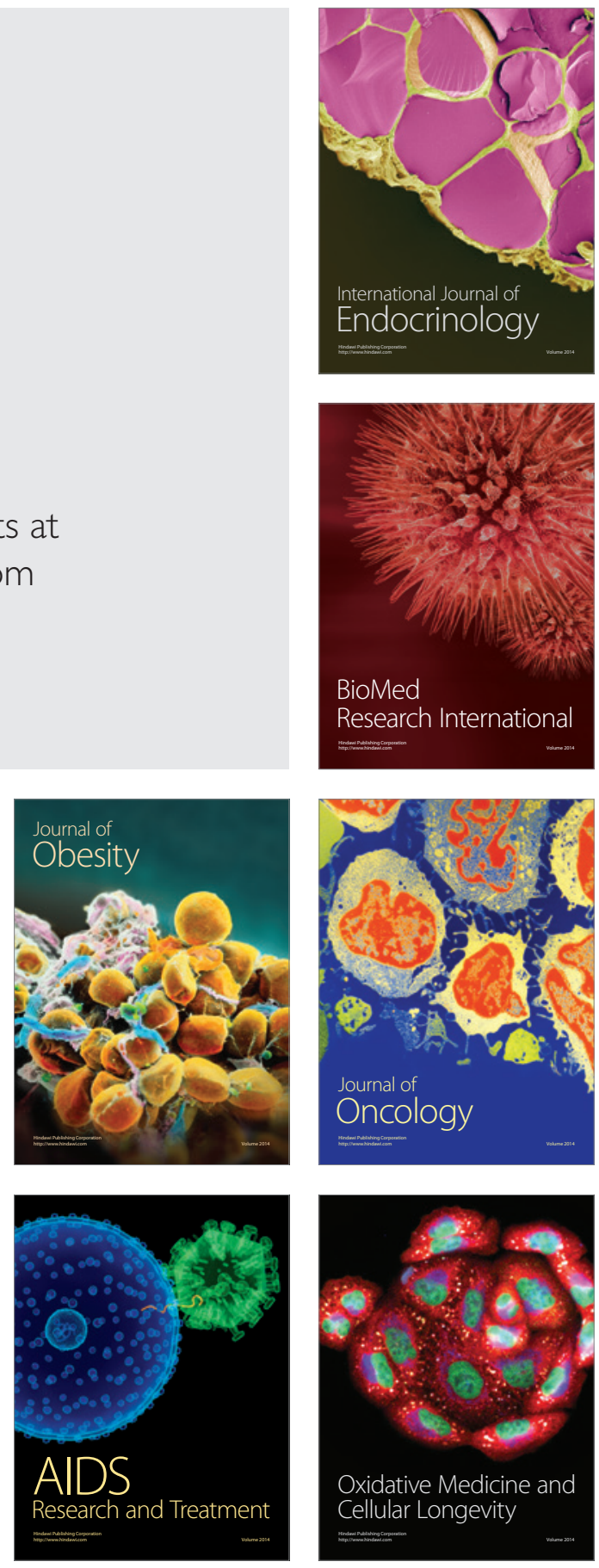